\title{
Efficacy of glimepiride on insulin resistance, adipocytokines, and atherosclerosis
}

\author{
Kunihiko Koshiba, Masahiro Nomura*, Yutaka Nakaya, and **Susumu Ito \\ Department of Digestive and Cardiovascular Medicine, ${ }^{*}$ Faculty of Integrated Art and Sciences, Department \\ of Human and Social Sciences, ${ }^{* *}$ Department of Nutrition and Metabolism, The University of Tokushima \\ Graduate School, Tokushima, Japan
}

\begin{abstract}
Background : Plasma adiponectin levels increase after the administration of glimepiride. This unique effects would also be expected to improve other adipocytokines and have antiatherosclerotic action in patients with metabolic syndrome.

Methods : Thirty-four patients with type 2 diabetes mellitus who were administrated glibenclamide were randomly divided into two groups. In 20 patients glibenclamide was changed to glimepiride (GP group), and the administration of glibenclamide (GB group) was continued in 14 patients. Twelve patients receiving insulin therapy (INS group) were enrolled for comparison. The levels of plasma adiponectin, high sensitive-CRP, TNF- $\alpha$, interleukin-6, homeostasis model assessment-insulin resistance (HOMA-IR), brachial-ankle pulse wave velocity (baPWV) and augmentation index (AI) were measured before and 28 weeks after the therapy.

Results: HOMA-IR in the GP group was significantly decreased compared to the GB group. Plasma adiponectin levels were significantly increased in the GP group but not in the other groups. TNF- $\alpha$, interleukin-6 and high sensitive-CRP levels were significantly decreased in the GP group, but not in the other groups. The baPWV and AI levels did not change in either the GB or the INS group, but were significantly decreased in the GP group. Conclusions:Glimepiride appears to improve insulin resistance and atherosclerotic disorders. J. Med. Invest. 53:87-94, February, 2006
\end{abstract}

Keywords : glimepiride, insulin resistance, adipocytokines, atherosclerosis

\section{INTRODUCTION}

The number of patients with type 2 diabetes mellitus world-wide has increased rapidly. The growing incidence of type 2 diabetes is due to life-style factors, i. e. increased caloric intake and decreased physical activity. Diabetes mellitus is considered to be mainly the result of an inadequate supply of insulin, i.e. decreased insulin secretion and insulin resistance. Sulfonylurea (SU)

Received for publication November 29, 2005 ; accepted December $26,2005$.

Address correspondence and reprint requests to Kunihiko Koshiba, M.D., Department of Digestive and Cardiovascular Medicine, University of Tokushima Graduate School, Kuramoto-cho, Tokushima 770-8503, Japan and Fax : +81-88-633-9235 derivatives which stimulates insulin secretion from $\beta$-cells of pancreas are widely used in the treatment of diabetes. Major complications associated with SU agents are hypoglycemia, weight gain and the exhaustion of beta $(\beta)$ cells with its hyper-insulinemic effect.

Insulin resistance ultimately involves the development of obesity, metabolic disorder, hypertension and atherosclerotic diseases. Therefore, concepts such as "syndrome X","deadly quartet", "visceral fat syndrome", "multiple risk factor syndrome" and "metabolic syndrome" are used to describe this situation, and comprehensive therapy that addresses these clinical conditions is required. Recent drug strategies are also targeted to improve insulin resistance rather than the promotion 
of insulin secretion.

Although glimepiride, a third-generation SU agent, has lesser insulin secretion action from the pancreatic $\beta$-cells compared with the conventional SU agent, its hypoglycemic action is equivalent to conventional SU agents (1). It has recently been reported that plasma adiponectin levels increase after the administration of glimepiride (2). Adiponectin shows anti-atherosclerotic effects on the vascular wall, and insulin sensitivity is increased in skeletal muscle and liver. The unique effects of this drug might be useful in patients with metabolic syndrome.

In the present study, plasma adiponectin, high sensitive-CRP (hs-CRP), tumor necrosis factor $-\alpha$ (TNF- $\alpha$ ), interleukin-6 (IL-6), homeostasis model assessment-insulin resistance (HOMA-IR), brachialankle pulse wave velocity (baPWV) and augmentation index (AI) were measured before and 28 weeks after the administration of glimepiride, and the effects of this diabetic drug on insulin resistance, inflammatory cytokines and anti-atherosclerotic effect were examined.

\section{PATIENTS AND METHODS}

\section{1) Patients}

Forty-six patients with type 2 diabetes mellitus (30 men and 16 women) participated in the present study. Patients who had coronary artery disease or arteriosclerosis obliterans were excluded from the study. Written informed consent was obtained from all subjects before their participation. Of 34 patients who were given glibenclamide, they were randomly divided into two groups. The medication for twenty patients (13 men and 7 women) was changed to glimepiride with their consent (GP group), and the other 14 patients ( 9 men and 5 women) underwent a continuous administration of glibenclamide (GB group). Twelve patients ( 8 men and 4 women) who were undergoing insulin therapy (INS group) were enrolled for comparison in the present study.

The patient characteristics of the present study are shown on Table 1 . There were no significant differences in age, systolic blood pressure, body mass index (BMI), fasting plasma glucose, glycohemoglobin (HbA1c), HOMA-IR between the GB and GP groups at the start of the study. The INS group was younger in comparison with the GB and GP groups $(\mathrm{p}<0.01)$, and fasting plasma glucose and $\mathrm{HbA1c}$ levels were significantly higher in INS group $(p<0.01)$.
Table 1. Patient profiles at baseline

\begin{tabular}{cccc}
\hline \hline Group & GB & GP & INS \\
\hline $\mathrm{n}($ case $)$ & 14 & 20 & 12 \\
gender(M/F) & $9 / 5$ & $13 / 7$ & $8 / 4$ \\
Age(year) & $71.7 \pm 6.2$ & $70.2 \pm 7.6$ & $65.6 \pm 8.2^{*}$ \\
$\mathrm{SBP}(\mathrm{mmHg})$ & $152 \pm 22$ & $148 \pm 26$ & $156 \pm 24$ \\
$\mathrm{BMI}\left(\mathrm{kg} / \mathrm{m}^{2}\right)$ & $24.2 \pm 2.2$ & $22.3 \pm 3.6$ & $23.4 \pm 6.2$ \\
$\mathrm{FPG}(\mathrm{mg} / \mathrm{dl})$ & $146 \pm 38$ & $152 \pm 22$ & $159 \pm 42^{*}$ \\
$\mathrm{HbA} 1 \mathrm{c}(\%)$ & $7.8 \pm 2.0$ & $7.7 \pm 1.9$ & $8.6 \pm 2.2^{*}$ \\
$\mathrm{HOMA}-\mathrm{IR}$ & $2.33 \pm 2.6$ & $2.41 \pm 2.9$ & - \\
\hline & & $*$ & $\mathrm{p}<0.01$ vs GB and GP group
\end{tabular}

\section{2) Change from glibenclamide to glimepiride}

According to a previous report by Inukai (3), glibenclamide is converted to glimepiride at a dose of glimepiride $1 \mathrm{mg}$ for glibenclamide $1.25 \mathrm{mg}$ in the GP group. Therefore, referring to plasma glucose levels, the glimepiride dosage was progressively decreased or increased up to maximal dose of $6 \mathrm{mg} /$ day. Pulse wave velocity, BMI and biochemical examinations were conducted before and 28 weeks after the administration of glimepiride

\section{3) Pulse wave velocity, BMI and biochemical examinations}

Blood pressure, pulse wave velocity and BMI were measured, and biochemical examinations, including fasting plasma glucose (FPG), immunoreactive insulin (IRI), adiponectin, hs-CRP, TNF- $\alpha$, IL-6 was carried out during the continuous therapy (GB and INS groups) before and 28 weeks after therapy (GP group).

After a 15-minute bed rest in a supine position, baPWV was measured using a sphygmomanometer and a sphygmograph device (FORM PWV/ABI, COLIN Medical Technology Co. Ltd., Tokyo, Japan). In addition to recording the limb lead ECG and phonocardiograms, mechanocardiograms were simultaneously recorded by attaching blood pressure cuffs with a tonometic sensor to the bilateral upper arms and ankles to measure the pulse transit time (PTT) of arterial waves from the right brachial artery to the ankle artery. Using these data, baPWV was obtained by dividing the length of blood vessels between the heart and inferior limb (L, which was obtained from the height using a regression formula) by PTT : baPWV=L / PTT $(\mathrm{cm} / \mathrm{sec})(4)$. In the present study, a higher value of baPWV, which was obtained at the bilateral arteries in the inferior limbs, was employed. Although values in which significant noise occurred were recalculated, the recording was successfully performed for all subjects.

The augmentation index (AI) was obtained by 
detecting carotid arterial waveforms using a carotid arterial sensor (CAP-350, COLIN Medical Technology Co. Ltd., Tokyo, Japan) and a multi-tonometric sensor. The systolic arterial waveforms consist of two different waves (percussion waves and tidal waves), and O'Rourke et al.(5) reported that the ratio of the differences in height between the tidal waves and percussion waves to the height of all waves was AI, an index of arteriosclerosis. AI may facilitate the evaluation of the severity of arterial wall sclerosis caused by endothelial cell dysfunction during the initial stage of arteriosclerosis (6).

BMI was obtained using the following equation. BMI= (body weight; $\mathrm{kg}) /(\text { height; } \mathrm{m})^{2}$. As an index of insulin resistance, HOMA-IR was obtained using the following equation. HOMA-IR $=\mathrm{FPG}(\mathrm{mg} / \mathrm{dl}) \times$ fasting IRI $(\mathrm{mU} / \mathrm{ml}) \div 405$

An enzyme immunoassay (BML Co. Ltd., Tokyo, Japan) was used to determine adiponectin, hs-CRP, TNF- $\alpha$ and IL- 6 .

\section{4) Statistical analysis.}

All values were expressed as the mean $\pm \mathrm{SD}$. Statistical analyses were performed using the Student's paired $t$-test (two-tail), and values of $p<0.05$ were considered to indicate statistical significance. The chi square test was used to examine differences with categorical variables. Data analysis was performed using the StatView statistical software (Stat View 5.0; SAS Institute, Inc., Cary, NC, USA).

\section{RESULTS}

\section{1) Blood pressure, BMI, FPG, HbAlc and HOMA-IR among GB, GP and INS groups.}

Table 2 shows data for blood pressure, BMI, FPG, HbA1c and HOMA-R for the GB, GP and INS groups 28 weeks after the start of the study. No significant differences in blood pressure and BMI were found among the groups. In a comparison of the GB group

Table 2. Patient profiles after 28 weeks

\begin{tabular}{cccc}
\hline \hline Group & GB & GP & INS \\
\hline SBP $(\mathrm{mmHg})$ & $148 \pm 24$ & $142 \pm 18$ & $150 \pm 26$ \\
$\mathrm{BMI}\left(\mathrm{kg} / \mathrm{m}^{2}\right)$ & $23.2 \pm 2.4$ & $22.0 \pm 3.6$ & $22.4 \pm 6.2$ \\
$\mathrm{FPG}(\mathrm{mg} / \mathrm{dl})$ & $142 \pm 32$ & $123 \pm 20^{*}$ & $146 \pm 32$ \\
HbA1c(\%) & $7.6 \pm 2.0$ & $7.1 \pm 1.9^{*}$ & $8.3 \pm 2.2^{* *}$ \\
HOMA-IR & $2.23 \pm 2.2$ & $1.88 \pm 1.1^{* * *}$ & - \\
\hline & & & $\mathrm{P}<0.01$ vs GB and INS group \\
& & $* *$ & $\mathrm{P}<0.01$ vs GB and GP group \\
& & $\mathrm{P}<0.01$ vs GB group
\end{tabular}

and INS group, FPG was significantly lower in the GP group $(\mathrm{p}<0.01)$. HbA1c and HOMA-IR in the GP group were significantly lower compared to GB group $(\mathrm{p}<0.01)$.

2) $P W V$ before and 28 weeks after glimepiride administration

Figure 1 shows a representative case of baPWV, AI, adiponectin, TNF- $\alpha$, IL- 6 and hs-CRP before and 28 weeks after glimepiride administration (73 year old male). The baPWV was decreased from $1930 \mathrm{~cm} / \mathrm{sec}$ to $1630 \mathrm{~cm} / \mathrm{sec}$ after the administration of glimepiride. The AI was also decreased from $28 \%$ to $18 \%$ after glimepiride administration. TNF- $\alpha$, interleukin- 6 and hs-CRP was decreased, and adiponectin was increased as the result of the glimepiride treatment.

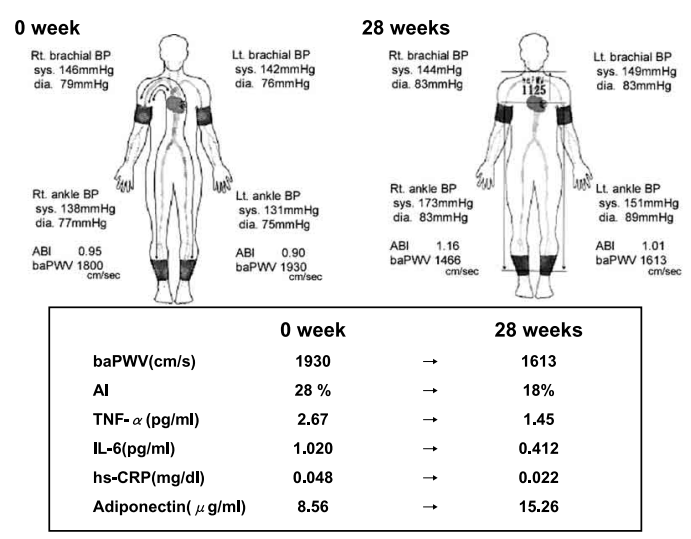

Fig. 1. Representative case of baPWV, AI, adiponectin, TNF- $\alpha$, IL- 6 and hs-CRP before and 28 weeks after glimepiride administration (73 year old male).

Rt;right, Lt;Left, sys;systolic, dia;diastolic, BP;blood pressure, ABI; Ankle Brachial Index, baPWV; brachial-ankle pulse wave velocity, $\mathrm{AI}$; Augmentation Index, TNF- $\alpha$; tumor necrosis factor- $\alpha$, IL- 6 ; interleukin-6, hs-CRP; high sensitive-CRP

\section{3) Change of inflammatory cytokines before and after therapy}

Changes of plasma adiponectin levels are shown in Figure 2. The treatment had no effect on plasma adiponectin levels in the GB and INS groups, but were significantly increased in the GP group $(\mathrm{p}<0.05)$. As shown in Figure 3, TNF- $\alpha$ levels did not significantly change in the GB and INS groups, but were significantly decreased in the GP group at the end of the study $(p<0.05)$. Figures 4 and 5 show that IL- 6 and hs-CRP, the same as TNF- $\alpha$, did not change significantly in the GB and INS groups. However, IL-6 and hs-CRP levels were significantly decreased in the GP group $(\mathrm{p}<0.05)$. 


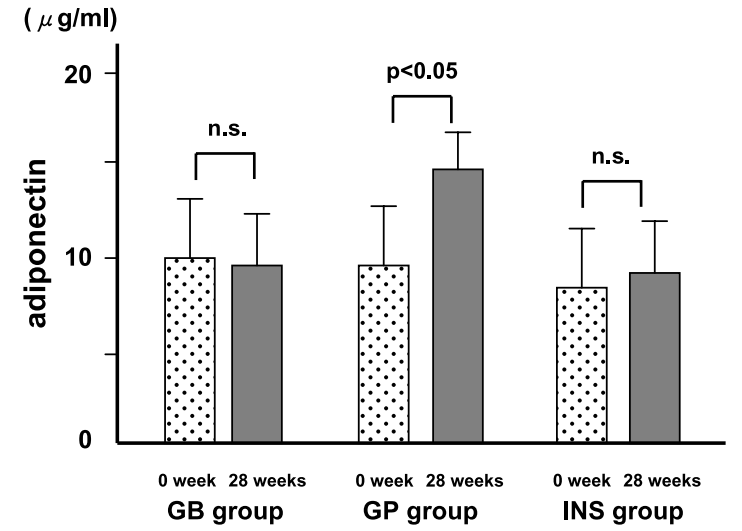

Fig.2. Changes in plasma adiponectin levels during continuous therapy (GB and INS groups) or before and 28 weeks after therapy (GP group).

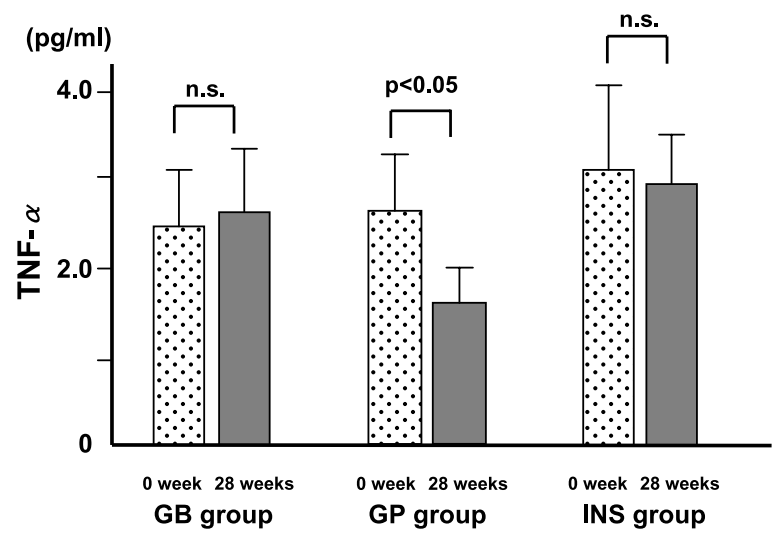

Fig. 3. Changes in TNF- $\alpha$ levels during continuous therapy (GB and INS groups) or before and 28 weeks after therapy (GP group). TNF- $\alpha$; tumor necrosis factor $-\alpha$.

\section{4) Changes of atherosclerotic parameters (baPWV/ $A B I$, augmentation index and HOMA-IR)}

Changes in atherosclerotic parameters for each group are shown in Figure 6. The baPWV did not change significantly in the GB and INS groups during the study period, but baPWV significantly decreased in the GP group at the end of the study $(\mathrm{p}<0.05)$. Changes in $\mathrm{AI}$ are shown in Figure 7. Similar to baPWV, AI levels did not significantly change during the study period in the GB and INS groups, but were significantly decreased in GP group ( $\mathrm{p}<0.05)$. HOMA-IR significantly decreased in the GP groups at the end of the study $(p<0.05)$, but did not significantly change during the study period in the GB group.

\section{DISCUSSION}

The present study confirms that, in comparing the GB group and INS groups, HOMA-IR was significantly lower in the GP group, suggesting that glimepiride has a stronger effect on improving insulin

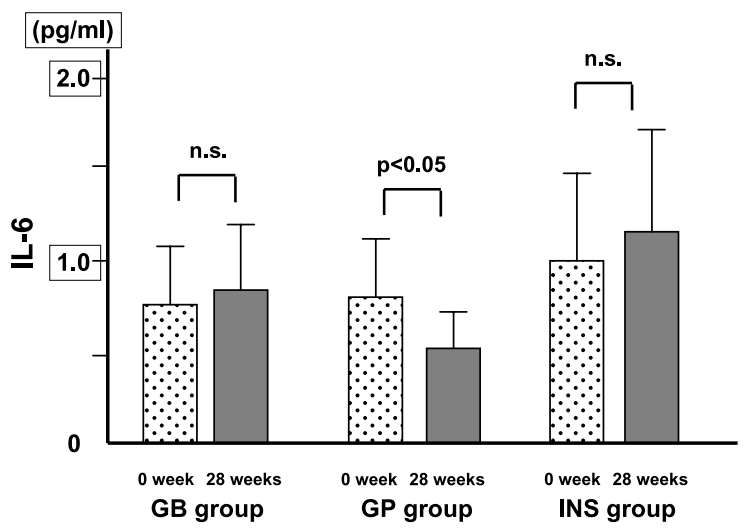

Fig. 4. Changes in IL-6 levels during continuous therapy (GB and INS groups) or before and 28 weeks after therapy (GP group). IL-6 ; interleukin-6.

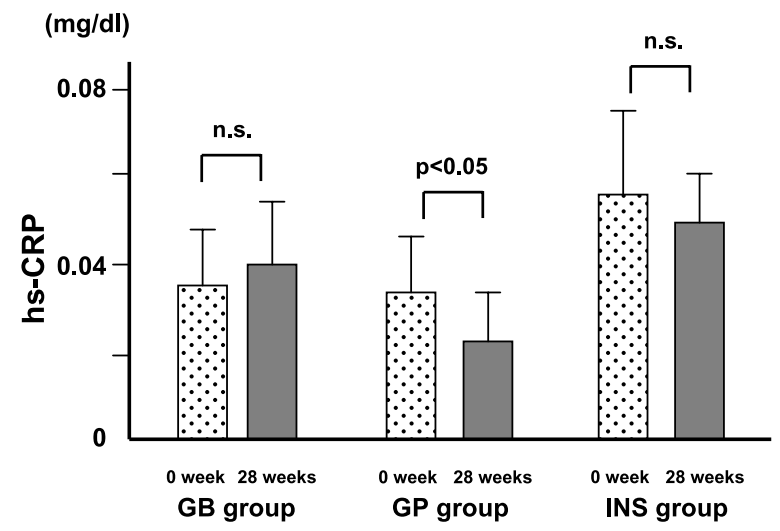

Fig. 5. Changes in hs-CRP levels during continuous therapy (GB and INS groups) or before and 28 weeks after therapy (GP group). hs-CRP; high sensitive-CRP.

resistance. Furthermore, adiponectin levels were significantly increased, and both inflammatory cytokines and atherosclerotic parameters (baPWV, $\mathrm{AI}$ ) decreased in the GP group. These results suggest that the administration of glimepiride would have a supressive effect on atherosclerosis.

Conventional SU agents stimulate insulin secretion from pancreatic $\beta$-cells, but they cannot reproduce the spike-shaped insulin secretion in response to a meal. As a result, delayed hyperinsulinemia promotes the accumulation of visceral fat. This suggests that conventional SU agents worsen insulin resistance and induce atherosclerosis (7).

Although glimepiride has a hypoglycemic effect equivalent to the conventional SU agents, its effect on insulin secretion is weak. Therefore, the findings here in present study suggest that glimepiride contributes to the improvement of hyperinsulinemia, visceral fat accumulation and atherosclerotic suppression. 


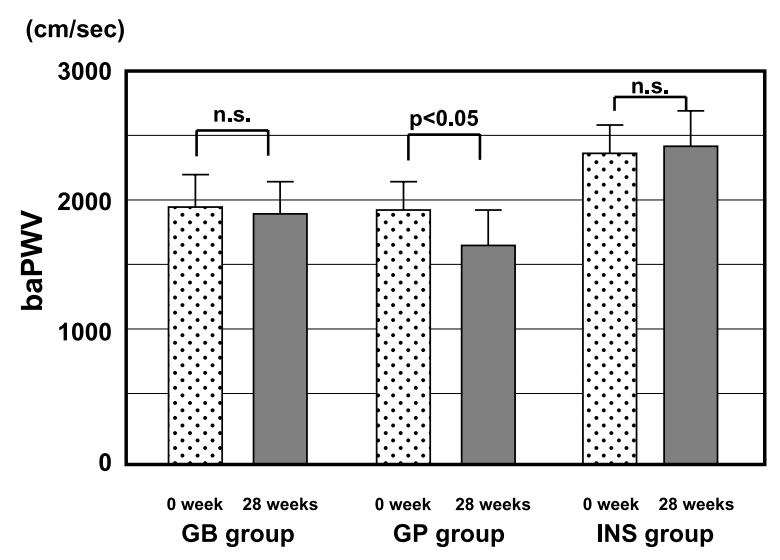

Fig. 6. Changes in the baPWV for each group during continuous therapy (GB and INS groups) or before and 28 weeks after therapy (GP group).

baPWV; brachial-ankle pulse wave velocity.

(\%)

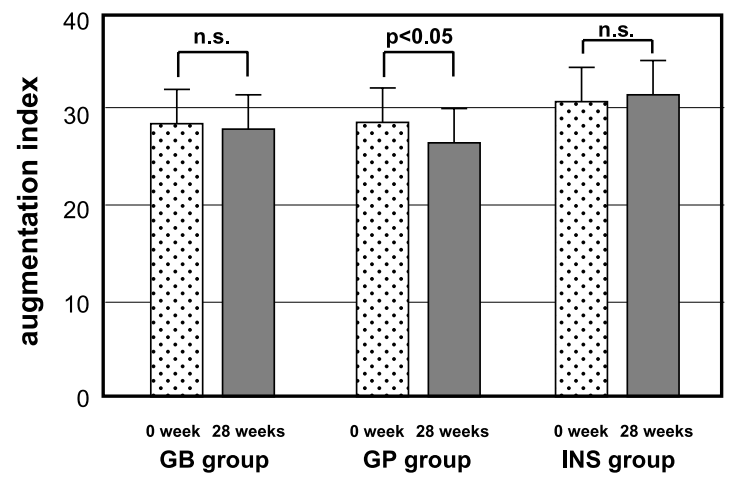

Fig. 7. Changes in $\mathrm{AI}$ levels during continuous therapy (GB and INS groups) or before and 28 weeks after therapy (GP group). $\mathrm{AI}$; augmentation index.

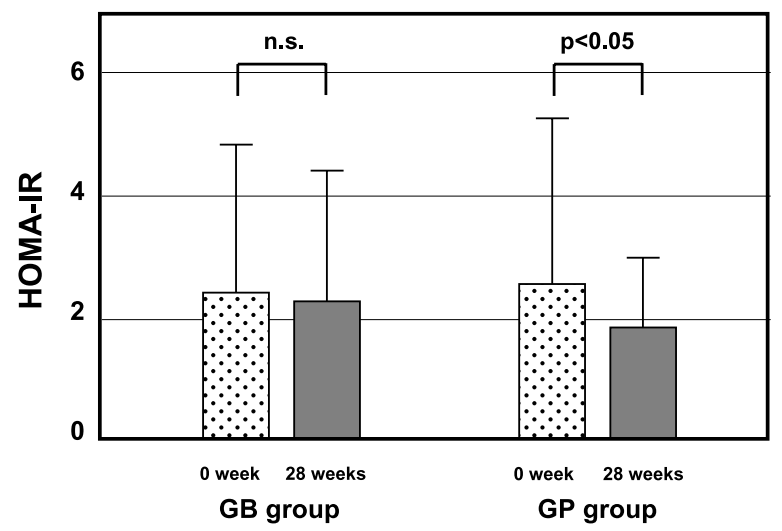

Fig. 8. Changes in HOMA-IR during continuous therapy (GB groups) or before and 28 weeks after therapy (GP group). HOMA-IR ; homeostasis model assessment-insulin resistance.

\section{1) Effects of glimepiride on insulin resistance}

In the present study, glimepiride was found to improve insulin resistance, consistent with results reported in previous studies. This effect appears to be based on the promotion of glucose-uptake in the liver and peripheral tissue, and the suppression of glucose release in the liver (8). It has been reported that glimepiride promotes the production of fluctose-2, 6-bisphosphate in the liver, promotes the glycolytic pathway, inhibits gluconeogenesis and glucose release, and suppresses ketogenesis in an in-vitro study (9).

Glimepiride also promotes glucose-uptake in skeletal muscle (10) and adipose tissue (11). Consistent with previous studies (2), we also found that increased adiponectin and decreased TNF- $\alpha$ levels. Adiponectin is an adipose tissue-specific glycoprotein that activates tyrosine kinase activity without connecting directly to insulin receptors, promotes the tyrosine phosphorylation of insulin receptor substrate-1 (IRS-1) and insulin receptor substrate-2 (IRS-2), and activates glycogen syntheses through various intracellular signals. In addition, Yamauchi et al. repoted that adiponectin increases phosphorylation and activity of the 5'-AMP-activated protein kinase, and that it simultaneously increases glucose uptake in myocytes and the liver, thereby directly regulates glucose metabolism and insulin sensitivity (12).

On the other hand, TNF- $\alpha$ decreases the tyrosine kinase activity of insulin receptors (13), reduces glucose transport, and induces insulin resistance (14). Adipocytokines, i. e. adiponectin and TNF- $\alpha$, inhibit and compete for each other during the biosynthetic phase in adipose tissue and in peripheral tissue (15).

The mechanism associated with the increase in adiponectin after glimepiride administration remains unclear. There are two biosynthetic pathways for the production of adiponectin, one is the direct production of adiponectin through Peroxisome ProliferatorActivated Receptor -gamma (PPAR- $\gamma$ ), an intranuclear receptor of adipocytes, and the other is the relative increase induced by the decrease in TNF- $\alpha$, which has an inhibitory effect on the production of adiponectin.

Recently, it has been reported that glimepiride has a direct action on PPAR- $\gamma$ (16). The maximum PPAR- $\gamma$ activity enhancing effect of glimepiride is approximately $20 \%$ that of 1 microM pioglitazone. In contrast, this PPAR- $\gamma$ stimulatory effect was not observed under the same conditions with glibenclamide(17). This might be the cause of the significant increase of adiponectin after glimepiride administration .

Adiponectin was increased and TNF- $\alpha$ and HOMA-IR were decreased significantly after the administration of glimepiride in the present study. HOMA-IR has reported to be positively correlated with an index of insulin resistance by a glucose clamp method (18), and HOMA-IR is an established index of insulin resistance. The present study reveals that glimepiride 
has a strong effect on improving insulin resistance in comparison with glibenclamide.

\section{2) Effects of glimepiride on body weight and BMI}

Mori et al. (19) reported that the expression of mRNA of TNF- $\alpha$ in retroperitoneal adipose tissue was significantly decreased in a glimepiride-administered group compared with a glibenclamide-administered group in an experimental study using rats as models. Further study will be required to investigate the reason for why TNF- $\alpha$ decreases and adiponectin increases after administration of glimepiride.

Some clinical researchers have also reported that neither weight nor BMI is increased after the administration of glimepiride $(20,21)$. In agreement with these studies, no significant change in BMI was found in the present study, and our results were in agreement with previous reports.

\section{3) Anti-atherosclerotic effect of glimepiride}

In the initial phases of atherosclerosis, injured vascular endothelial cells express intercellular adhesion molecules, such as vascular cell adhesion molecule-1 (VCAM-1), intracellular adhesion molecule - 1(ICAM-1) and E-secretin, and monocytes became attached to the vascular wall. Monocytes invade the vascular endothelium, secrete cytokines such as TNF- $\alpha$, accumulate cholesterol esters and are transformed into foam cells. Furthermore, TNF- $\alpha$ induces the further adhesion of monocytes. At the injured endothelium, adiponectin suppresses the expression of these intercellular adhesion molecules, the levels of which increase in a TNF- $\alpha$-dependent manner (22), suppresses the expression and secretion of TNF- $\alpha(23)$, suppresses the expression of class A scavenger receptors and inhibits the foaming of macrophages (24). These mechanisms inhibit both the initiation and the development of atherosclerosis.

Glimepiride leads to an improvement in lipid metabolism (25), improves endothelial function due to the biosynthesis of nitric monoxide (26), and has anti-oxidative effects (26) in addition to the antiatherosclerotic effects $(26,27)$ mediated by these cytokines. The present study revealed that biochemical parameters (IL-6, hs-CRP) significantly decreased, and pulse wave velocity parameters (baPWV, AI) also significantly decreased after the administration of glimepiride. These results suggest that glimepiride has an anti-atherosclerotic effect. The AHA/ACC recommendation in 2003 (28) reported the utility of hs-CRP measurements for the primary prevention of cardiovascular diseases. In the present study, a significant decrease in hs-CRP was found after glimepiride administration, and this drug contributes to the prevention of coronary heart disease.

The present study suggests that one of the effects of glimepiride is to improve insulin resistance, but that it has less possibility for preventing body weight. Furthermore, glimepiride also has an inhibitory effect on the initiation and development of atherosclerosis. Inukai et al. (3) reported that patients who had been treated with only SU, or treated with SU for a short time (less than 5 years), and who were obese (BMI $\geqq 25$ ) or had a high HOMA-IR(HOMA-IR $\geqq 3$ ), showed significantly reduced insulin resistance, such factors are important for predicting therapeutic effects when the use of glibenclamide is changed to glimepiride. In conclusion, the present study revealed that the use of glimepiride as a drug would be expected to improve insulin resistance and atherosclerotic disorders without a gain in body weight.

\section{STUDY LIMITATION}

In the present study, the improvement of an arteriosclerotic index by glimepride could not deny influence by improvement of glycemic control. In a future study, this problem could be solved by excluding the influence of improvement of an arteriosclerotic index by blood glucose improvement by crossover method.

\section{REFERENCES}

1. Draeger KE, Wernicke-Panten K, Lomp HJ, Schuler E, Rosskamp R : Long-term treatment of type 2 diabetic patients with the new oral antidiabetic agent glimepiride(Amaryl): a doubleblind comparison with glibenclamide. Horm Metab Res $28: 419-425,1996$

2. Tsunekawa T, Hayashi T, Suzuki Y, Matsui-Hirai H, Kano H, Fukatsu A, Nomura N, Miyazaki A, Iguchi A : Plasma adiponectin plays an important role in improving insulin resistance with glimepiride in elderly type 2 diabetic subjects. Diabetes Care 26 : 285-289, 2003

3. Inukai K, Watanabe M, Nakashima Y, Sawa T, Takata N, Tanaka M, Kashiwabara H, Yokota K, Suzuki M, Kurihara S, Awata T, Katayama S : Efficacy of glimepiride in Japanese type 2 diabetic subjects. Diabetes Res Clin Pract 68:250-257, 2005

4. Lehmann ED : Noninvasive measurements of 
aortic stiffness: methodological considerations. Pathol Biol (Paris) 47 : 716-730, 1999

5. O'Rourke MF, Mancia G : Arterial stiffness. J Hypertens 17 : 1-4, 1999

6. Harada S, Takeda K:Pulse wave velocity (PWV). Nippon Rinsho 62 (in Japanese) : 1136-1142, 2004

7. Purnell JQ, Weyer C: Weight effect of current and experimental drugs for diabetes mellitus : from promotion to alleviation of obesity. Treat Endocrinol $2: 33$ - 47, 2003

8. Muller G, Satoh Y, Geisen K: Extrapancreatic effects of sulfonylureas--a comparison between glimepiride and conventional sulfonylureas. Diabetes Res Clin Pract 28(Suppl):S115-137, 1995

9. Hatao K, Kaku K, Matsuda M, Tsuchiya M, Kaneko T:Sulfonylurea stimulates liver fructose-2, 6-bisphosphate formation in proportion to its hypoglycemic action. Diabetes Res Clin Pract 1 : 49-53, 1985

10. Takada $Y$, Takata $Y$, Iwanishi $M$, Imamura $T$, Sawa T, Morioka H, Ishihara H, Ishiki M, Usui I, Temaru R, Urakaze M, Satoh Y, Inami T, Tsuda S, Kobayashi M: Effect of glimepiride (HOE 490) on insulin receptors of skeletal muscles from genetically diabetic KK-Ay mouse. Eur J Pharmacol 308 : 205-210, 1996

11. Muller G, Wied S, Wetekam EM, Crecelius A, Unkelbach A, Punter J: Stimulation of glucose utilization in 3T3 adipocytes and rat diaphragm in vitro by the sulphonylureas, glimepiride and glibenclamide, is correlated with modulations of the cAMP regulatory cascade. Biochem Pharmacol 48 : 985-996, 1994

12. Yamauchi T, Kamon J, Minokoshi Y, Ito Y, Waki H, Uchida S, Yamashita S, Noda M, Kita S, Ueki K, Eto K, Akanuma Y, Froguel P, Foufelle F, Ferre P, Carling D, Kimura S, Nagai R, Kahn BB, Kadowaki T: Adiponectin stimulates glucose utilization and fatty-acid oxidation by activating AMP-activated protein kinase. Nat Med 8:12881295, 2002

13. Ozes ON, Akca H, Mayo LD, Gustin JA, Maehama T, Dixon JE, Donner DB : A phosphatidylinositol 3-kinase/Akt/mTOR pathway mediates and PTEN antagonizes tumor necrosis factor inhibition of insulin signaling through insulin receptor substrate-1. Proc Natl Acad Sci USA 98 : 4640-4645, 2001

14. Jain R, Police S, Phelps K, Pekala PH:Tumour necrosis factor-alpha regulates expression of the CCAAT-enhancer-binding proteins (C/EBPs) alpha and beta and determines the occupation of the $\mathrm{C} / \mathrm{EBP}$ site in the promoter of the insulin- responsive glucose-transporter gene in 3 T3-L 1 adipocytes. Biochem J 338 (Pt 3):737-743, 1999

15. Ouchi N, Kihara S, Arita $Y$, Maeda K, Kuriyama H, Okamoto Y, Hotta K, Nishida M, Takahashi M, Nakamura T, Yamashita S, Funahashi T, Matsuzawa Y : Novel modulator for endothelial adhesion molecules:adipocyte-derived plasma protein adiponectin. Circulation 100:2473-2476, 1999

16. Fukuen S, Iwaki M, Yasui A, Makishima M, Matsuda M, Shimomura I: Sulfonylurea agents exhibit peroxisome proliferator-activated receptor gamma agonistic activity. J Biol Chem 280 : 2365323659, 2005

17. Inukai K, Watanabe M, Nakashima Y, Takata N, Isoyama A, Sawa T, Kurihara S, Awata T, Katayama S: Glimepiride enhances intrinsic peroxisome proliferator-activated receptorgamma activity in 3T3-L1 adipocytes. Biochem Biophys Res Commun 328 : 484-490, 2005

18. Emoto M, Nishizawa $Y$, Maekawa K, Hiura $Y$, Kanda H, Kawagishi T, Shoji T, Okuno Y, Morii $\mathrm{H}$ : Homeostasis model assessment as a clinical index of insulin resistance in type 2 diabetic patients treated with sulfonylureas. Diabetes Care 22 : 818-822, 1999

19. Mori Y, Komiya H, Kurokawa N, Tajima N : Comparison of the effects of glimepiride and glibenclamide on adipose tissue tumour necrosis factor-alpha mRNA expression and cellularity. Diabetes Obes Metab $6: 28-34,2004$

20. Martin S, Kolb H, Beuth J, van Leendert R, Schneider B, Scherbaum WA: Change in patients' body weight after 12 months of treatment with glimepiride or glibenclamide in Type 2 diabetes: a multicentre retrospective cohort study. Diabetologia 46 : 1611-1617, 2003

21. Weitgasser R, Lechleitner M, Luger A, Klingler A: Effects of glimepiride on $\mathrm{HbA}(1 \mathrm{c})$ and body weight in Type 2 diabetes: results of a 1.5-year follow-up study. Diabetes Res Clin Pract 61 : 13-19, 2003

22. Ouchi N, Kihara S, Arita Y, Okamoto Y, Maeda K, Kuriyama H, Hotta K, Nishida M, Takahashi M, Muraguchi M, Ohmoto Y, Nakamura T, Yamashita S, Funahashi T, Matsuzawa Y : Adiponectin, an adipocyte-derived plasma protein, inhibits endothelial NF-kappaB signaling through a cAMPdependent pathway. Circulation 102:1296-1301, 2000

23. Yokota T, Oritani K, Takahashi I, Ishikawa J, Matsuyama A, Ouchi N, Kihara S, Funahashi T, 
Tenner AJ, Tomiyama Y, Matsuzawa Y: Adiponectin, a new member of the family of soluble defense collagens, negatively regulates the growth of myelomonocytic progenitors and the functions of macrophages. Blood $96: 1723-1732,2000$

24. Ouchi N, Kihara S, Arita Y, Nishida M, Matsuyama A, Okamoto Y, Ishigami M, Kuriyama H, Kishida K, Nishizawa H, Hotta K, Muraguchi M, Ohmoto Y, Yamashita S, Funahashi T, Matsuzawa Y:Adipocytederived plasma protein, adiponectin, suppresses lipid accumulation and class A scavenger receptor expression in human monocyte-derived macrophages. Circulation 103 : 1057-1063, 2001

25. Derosa G, Franzetti I, Gadaleta G, Ciccarelli L, Fogari R: Metabolic variations with oral antidiabetic drugs in patients with Type 2 diabetes: comparison between glimepiride and metformin. Diabetes Nutr Metab $17: 143-150,2004$

26. Ueba H, Kuroki M, Hashimoto S, Umemoto T, Yasu T, Ishikawa S, Saito M, Kawakami M : Glimepiride induces nitric oxide production in human coronary artery endothelial cells via a PI3-kinase-Akt dependent pathway. Atherosclerosis $183: 35-39,2005$

27. Yang B, Tian H, Ren Y, Tong N, Yu H, Han L, Ran X : The change of atherogenic index of plasma (AIP) level in type 2 diabetic pedigrees and the response of AIP to Acarbose or Glimepiride in therapy of type 2 diabetes mellitus. Sheng Wu Yi Xue Gong Cheng Xue Za Zhi 22 (English abstract) : 560-4, 2005

28. Pearson TA, Mensah GA, Alexander RW, Anderson JL, Cannon RO 3rd, Criqui M, Fadl YY, Fortmann SP, Hong Y, Myers GL, Rifai N, Smith SC Jr, Taubert K, Tracy RP, Vinicor F : Centers for Disease Control and Prevention; American Heart Association. : Markers of inflammation and cardiovascular disease:application to clinical and public health practice:A statement for healthcare professionals from the Centers for Disease Control and Prevention and the American Heart Association. Circulation 107:499-511, 2003 